\title{
Elaboração e Validação de Escala Diagramática para Avaliação da Mancha-de-Cercospora em Melancia
}

\author{
Bernardo A. Halfeld-Vieira \& Kátia L. Nechet \\ Embrapa Roraima, BR 174, km 08, Cx. Postal 133, CEP 69301-970, Boa Vista, RR, fax (95) 626-7125, \\ e-mail: halfeld@cpafrr.embrapa.br
}

(Aceito para publicação em 13/10/2005)

Autor para correspondência: Bernardo A. Halfeld-Vieira

HALFELD-VIEIRA, B.A. \& NECHET, K.L. Elaboração e validação de escala diagramática para avaliação da manchade-cercospora em melancia. Fitopatologia Brasileira 31:046-050. 2006.

\section{RESUMO}

Com a finalidade de propor métodos padronizados para avaliação da severidade da mancha-de-cercospora em folhas de melancia (Citrullus lanatus), foi desenvolvida uma escala diagramática considerando os seguintes valores de área necrosada: 2; 4; 8; 12; 27; 45; 71 e 93\%. A validação da escala foi realizada por dez avaliadores que estimaram a severidade de 50 folhas com diferentes intensidades de doença. Por meio de regressão linear, confrontando valores de severidade reais e estimados, analisou-se a acurácia e precisão dos avaliadores que utilizaram a escala diagramática. A repetibilidade dos resultados foi verificada em uma segunda avaliação sete dias após a primeira. Constatou-se acurácia e precisão das estimativas visuais efetuadas com o auxílio da escala diagramática. O coeficiente de determinação regressão foi em média superior a 0,94 e os erros absolutos concentrados dentro de limites aceitáveis. Os avaliadores mostraram boa repetibilidade das estimativas. A escala proposta mostrou-se adequada para avaliação da severidade da mancha-de-cercospora em melancia.

Palavras-chave adicionais: Citrullus lanatus, Cercospora citrullina, Cercospora apii s.lat., patometria.

\begin{abstract}
Development and validation of a diagrammatic scale to evaluate cercospora leaf spot in watermelon

To propose customized methods of evaluating the severity of cercospora leaf spot in watermelon (Citrullus lanatus) leaves, a diagrammatic scale was developed with necrotic area of $2 ; 4 ; 8 ; 12 ; 27 ; 45 ; 71$ and $93 \%$. For validation, the severity of 50 leaves presenting different intensities of the disease was estimated by ten raters. Accuracy and precision were determined by linear regression, relating assessments performed by raters using the scale to actual severity. Repetition of the results provided by raters was verified in a second evaluation, seven days after the first assessment. The scale provided good levels of accuracy and precision of visual estimates. The coefficient determination average was higher than 0.94, and absolute errors were lower than acceptable limits. Overall, the raters exhibited good repetition of estimates. The proposed diagrammatic scale proved to be adequate for severity assessments of cercospora leaf spot in watermelon.
\end{abstract}

Additional keywords: Citrullus lanatus, Cercospora citrullina, Cercospora apii s.lat., pathometry.

\section{INTRODUÇÃO}

A mancha-de-cercospora em melancia [Citrullus lanatus (Thunb.) Matsum. \& Nakai] é causada por Cercospora citrullina Cooke, patógeno que tem uma ampla gama de hospedeiros na família das cucurbitáceas. Juntamente com outras espécies de Cercospora, tem sido proposto que seja incorporado ao complexo Cercospora apii Fresen. s.lat. (Crous \& Braun, 2003). Em Roraima, a mancha-de-cercospora tem sido observada em plantios comerciais de melancia como a doença mais freqüente da cultura, em alguns casos, em alta severidade (Halfeld-Vieira et al. 2004a; Halfeld-Vieira et al. 2004b). Entretanto, há poucos relatos de danos significativos, como no Piauí (Athayde Sobrinho \& Menezes, 1997), fazendo com que seja considerada uma doença de pouca importância nas demais regiões produtoras (Rêgo \& Carrijo, 2000; Kurozawa et al., 2005). Para realização de estudos epidemiológicos que possam resultar em uma melhor compreensão desse patossistema, assim como na proposição de estratégias de controle eficazes, são necessários subsídios para a quantificação da doença. Para isso, é importante que hajam métodos padronizados para determinação da severidade da mancha-de-cercospora em plantas de melancia. As escalas diagramáticas são utilizadas para esta finalidade por serem práticas e com aplicação em diferentes condições, melhorando a acurácia e precisão do avaliador (Spósito et al., 2004). Considerando a inexistência de métodos adequados para quantificação da mancha-de-cercospora em melancia, é proposta uma escala diagramática para auxiliar na avaliação da severidade da doença, descrevendo-se como foi desenvolvida e validada. 


\section{MATERIAL E MÉTODOS}

\section{Elaboração da escala diagramática}

Foram coletadas 105 folhas de melancia das cultivares Charleston Gray e Crimson Sweet em plantio experimental em que se observou alta intensidade da mancha-de-cercospora. Esta foi a única doença detectada. As folhas com diferentes graus de severidade foram digitalizadas individualmente em scanner Genius ${ }^{\circledR}$, modelo ColorPage-Vivid 4, em resolução de 118,11 pixels. $\mathrm{cm}^{-1}$. Com o auxílio do programa Adobe ${ }^{\circledR}$ Photoshop ${ }^{\circledR} 6.0$ (Adobe Systems Incorporated, 2000), determinou-se o percentual de área necrótica, sem considerar o halo amarelo. Baseandose na lei de Weber-Fechner (Horsfall \& Cowling, 1978; Nutter Jr. \& Schultz, 1995) de acuidade visual foi elaborada uma escala diagramática com oito níveis de severidade, considerando a forma e distribuição das lesões observadas com maior freqüência.

\section{Validação da escala diagramática}

Para os testes de validação da escala diagramática foram selecionadas 50 imagens de folhas digitalizadas, abrangendo diferentes graus de severidade (Michereff et al., 2000; Leite \& Amorim, 2002; Spósito et al., 2004). As imagens foram inseridas aleatoriamente em slides individuais para visualização no Microsoft ${ }^{\circledR}$ PowerPoint $^{\circledR}$ 2000 (Microsoft Corporation, 1999). Dez avaliadores, somente um com experiência na quantificação de doenças e outro de danos de insetos, receberam uma cópia colorida impressa da escala diagramática, estimando a severidade da doença para cada folha. Não foi realizado treinamento prévio. Para verificar a repetibilidade das estimativas, a ordem da apresentação das imagens foi modificada e os mesmos avaliadores repetiram o processo com um intervalo de uma semana entre as duas avaliações. Regressões lineares foram elaboradas confrontando os valores reais de severidade com os dados das estimativas de cada avaliador, determinando-se o intercepto $(a)$, o coeficiente angular da reta $(b) \mathrm{e}$ o coeficiente de determinação da regressão $\left(R^{2}\right)$. A acurácia foi verificada por meio do teste $t$ averiguando as hipóteses Ho: $a=0$ e Ho: $b=1$, ao nível de significância $P=0,01$. A repetibilidade das estimativas por um mesmo avaliador foi determinada pelos mesmos parâmetros, obtidos nas regressões em que se considerou a primeira avaliação em relação à segunda (Nutter Jr. et al., 1993). As análises de regressão foram realizadas com o programa Microsoft ${ }^{\circledR}$ Excel 2000 (Microsoft Corporation, 1999).

\section{RESULTADOS E DISCUSSÃO}

Foi observado que, em condições naturais, o valor máximo de severidade da mancha-de-cercospora em melancia atinge $100 \%$, permanecendo as folhas presas à planta. Entretanto verifica-se com maior freqüência valores de até $42 \%$. Os sintomas da doença foram constatados não somente em folhas totalmente expandidas, mas também em fase de desenvolvimento. $\mathrm{O}$ halo amarelado nem sempre ocorreu ao redor das manchas.

A escala diagramática foi representada com valores de área foliar necrosada iguais a 2; 4; 8; 12; 27; 45; 71 e 93\% (Figura 1). A acurácia das estimativas dos avaliadores, indicativo da proximidade entre valores estimados e reais (Nutter Jr. et al., 1991), foi verificada considerando-se que valores de intercepto $(a)$ significativamente diferentes de
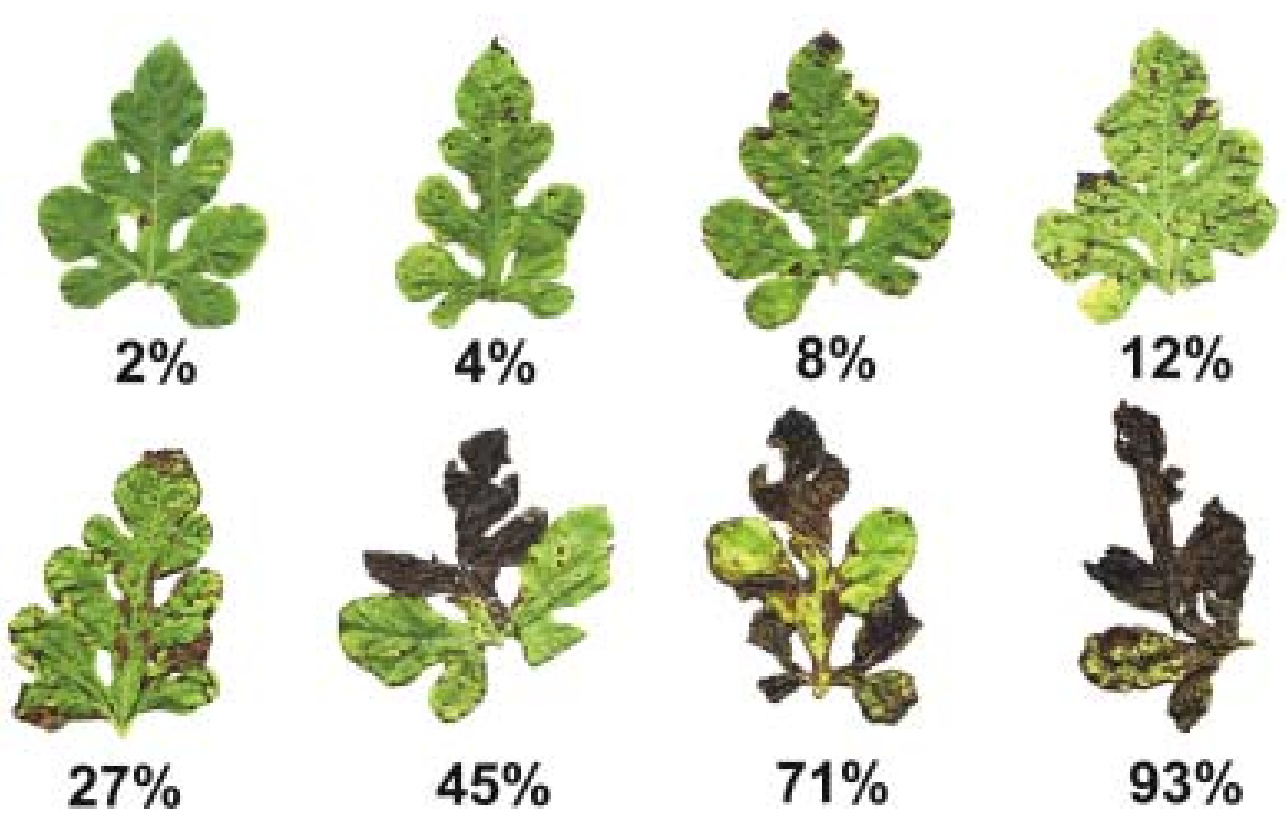

FIG. 1 - Escala diagramática para avaliação da severidade da mancha-de-cercospora em melancia (Citrullus lanatus). Os valores representam a porcentagem da área foliar necrosada. 
zero são indicativos de desvios constantes e valores de coeficiente angular (b) significativamente diferentes de 1 , indicam haver desvios sistemáticos. A precisão das estimativas foi averiguada pelo coeficiente de determinação da regressão $\left(R^{2}\right)$, variância dos erros absolutos e repetibilidade das estimativas por um mesmo avaliador (Leite \& Amorim, 1992; Nutter Jr. et al., 1993; Michereff et al., 2000).

As análises para validação da escala diagramática mostram que na primeira avaliação, os valores do intercepto para sete avaliadores não diferiram significativamente de zero (Tabela 1 e Figura 2). Para todos os avaliadores o coeficiente angular foi significativamente igual a 1 , não incorrendo em erros sistemáticos. Na segunda avaliação, os valores do intercepto para nove avaliadores não diferiu significativamente de zero e o coeficiente angular foi significativamente igual a $1(P \leq 0,01)$ para seis deles. Para cada $1 \%$ de incremento nos valores reais de severidade da mancha-de-cercospora verifica-se um aumento médio de $0,96 \%$ nos valores estimados pelos avaliadores, tanto na primeira como na segunda avaliação. Isto indica uma pequena tendência à subestimativa, embora os avaliadores C, E e G tenham superestimado significativamente a severidade da mancha-de-cercospora na primeira avaliação e apenas o avaliador C, na segunda avaliação (Tabela 1 e Figura 2). Outros estudos de validação de escalas diagramáticas indicam que a tendência em superestimar valores é mais freqüente (Parker et al., 1995; Díaz et al., 2001; Leite \& Amorim, 2002; Spósito et al., 2004).

Foi verificada alta precisão das estimativas visuais efetuadas com o auxílio da escala diagramática. Na primeira

TABELA 1 - Intercepto (a), coeficiente angular da reta $(b)$ e coeficiente de determinação $\left(R^{2}\right)$ das equações de regressão linear relacionando os valores reais e as estimativas de severidade da mancha-de-cercospora da melancia (Citrullus lanatus) efetuadas por dez avaliadores

\begin{tabular}{|c|c|c|c|c|c|c|}
\hline \multirow{2}{*}{ Avaliador } & \multicolumn{3}{|c|}{$1^{\mathrm{a}}$ avaliação } & \multicolumn{3}{|c|}{$2^{\mathrm{a}}$ avaliação } \\
\hline & $a$ & $b$ & $R^{2}$ & $a$ & $b$ & $R^{2}$ \\
\hline A & 0,55 & 0,96 & 0,95 & 0,91 & 1,04 & 0,94 \\
\hline B & 0,08 & 0,98 & 0,96 & 0,35 & 1,02 & 0,96 \\
\hline $\mathrm{C}$ & $4,15^{*}$ & 0,97 & 0,96 & $3,00 *$ & 0,98 & 0,95 \\
\hline $\mathrm{D}$ & 1,52 & 0,94 & 0,93 & 0,86 & 0,97 & 0,95 \\
\hline E & $2,92 *$ & 0,96 & 0,95 & 1,29 & $0,93^{*}$ & 0,97 \\
\hline $\mathrm{F}$ & 1,83 & 0,96 & 0,97 & 1,62 & $0,91 *$ & 0,96 \\
\hline G & $5,02 *$ & 1,03 & 0,94 & 0,05 & $0,87^{*}$ & 0,92 \\
\hline $\mathrm{H}$ & $-0,09$ & 0,94 & 0,94 & $-0,82$ & 0,97 & 0,95 \\
\hline I & 0,79 & 0,91 & 0,94 & $-1,06$ & 0,98 & 0,93 \\
\hline $\mathrm{J}$ & 0,32 & 0,97 & 0,95 & 1,74 & $0,89 *$ & 0,91 \\
\hline Média & 1,71 & 0,96 & 0,95 & 0,79 & 0,96 & 0,94 \\
\hline
\end{tabular}

*Asterisco representa situações em que a hipótese de nulidade ( $a=0$ ou $b=1$ ) foi rejeitada pelo teste $t(P \leq 0,01)$.

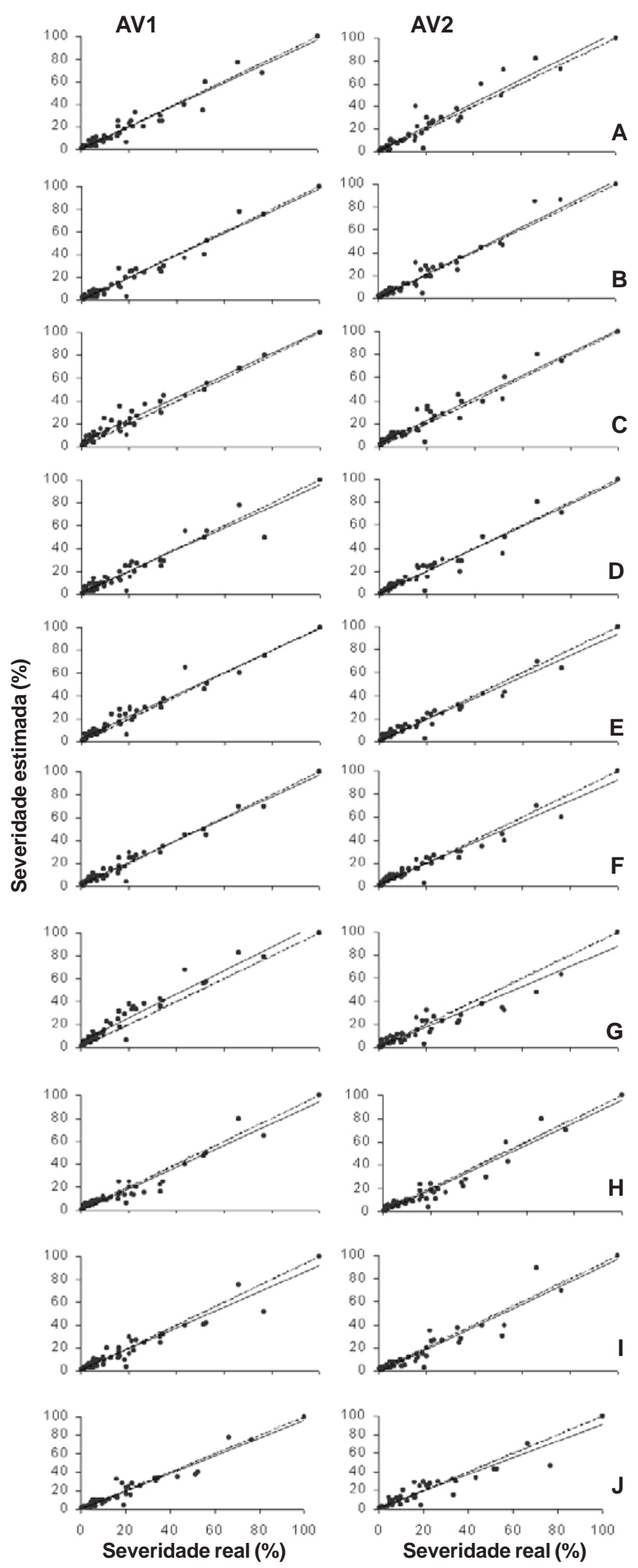

FIG. 2 - Severidade estimada $(\bullet)$ com o auxílio da escala diagramática para mancha-de-cercospora em melancia (Citrullus lanatus) na primeira (AV 1) e segunda (AV 2) avaliação, pelos dez avaliadores (A-J). A regressão linear, considerando valores de severidade real e estimada, está representada por linhas cheias. As linhas tracejadas representam o ajuste ideal em que a severidade real é igual à estimada. 
avaliação o coeficiente de determinação da regressão variou de 0,93 a 0,97 (média=0,95) e na segunda avaliação a variação verificada foi de 0,91 a 0,97 (média $=0,94$ ) (Tabela 1). Estes valores muitas vezes foram superiores aos obtidos em outros estudos (Nutter Jr. et al., 1993; Michereff et al., 2000; Spósito et al., 2004) e estão próximos a 0,95, considerado limiar desejável por Kranz (1988).

Os erros absolutos observados na análise de resíduos, que consideram a diferença entre valores absolutos de severidade estimada e real, confirmam a precisão dos avaliadores tanto na primeira quanto na segunda avaliação. Observa-se que a maioria dos erros absolutos ficou em níveis aceitáveis (Figura 3) conforme critérios adotados por outros autores (Michereff et al. 2000; Leite et al., 2002; Spósito et al., 2004).

Verificou-se boa reprodutibilidade das estimativas, em que a quantidade média de variação da primeira avaliação explicada pela segunda foi de 96\% (Tabela 2). Somente para dois avaliadores ( $F$ e G), os valores do coeficiente angular foram significativamente diferentes de 1 $(P \leq 0,01)$ (Tabela 2).

A escala diagramática proposta para avaliação da severidade da mancha-de-cercospora em melancia mostrou ser de fácil utilização, mesmo por pessoas sem nenhum treinamento em quantificação de doenças, proporcionando boa acurácia e precisão das estimativas.

\section{AGRADECIMENTOS}

Os autores agradecem a todos os avaliadores que contribuíram para validação da escala diagramática.

TABELA 2 - Intercepto (a), coeficiente angular da reta $(b)$ e coeficiente de determinação $\left(R^{2}\right)$ das equações de regressão linear relacionando a primeira à segunda estimativa da mancha-decercospora da melancia (Citrullus lanatus), por um mesmo avaliador

\begin{tabular}{cccc}
\hline \hline Avaliador & $\boldsymbol{a}$ & $\boldsymbol{b}$ & $\boldsymbol{R}^{\mathbf{2}}$ \\
\hline A & 0,60 & 1,07 & 0,96 \\
B & 0,38 & 1,04 & 0,99 \\
C & $-0,92$ & 1,00 & 0,96 \\
D & $-0,04$ & 0,99 & 0,95 \\
E & $-0,91$ & 0,93 & 0,95 \\
F & $-0,13$ & $0,95^{*}$ & 0,99 \\
G & $-3,18$ & $0,81^{*}$ & 0,89 \\
H & $-0,53$ & 1,01 & 0,98 \\
I & $-1,46$ & 1,05 & 0,94 \\
J & 1,68 & 0,91 & 0,94 \\
\hline Média & $-0,69$ & 0,98 & 0,96
\end{tabular}

* representa situações em que a hipótese de nulidade ( $a=0$ ou $b=1$ ) foi rejeitada pelo teste $t(P<0,01)$.
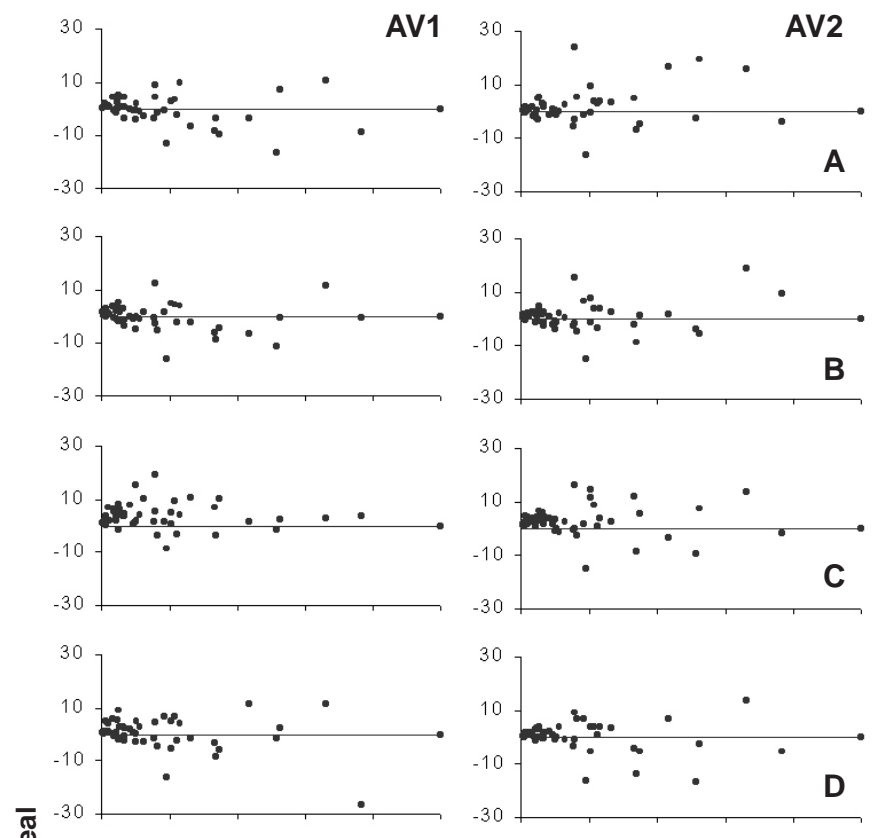

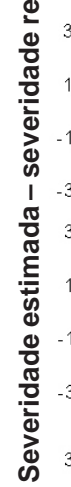
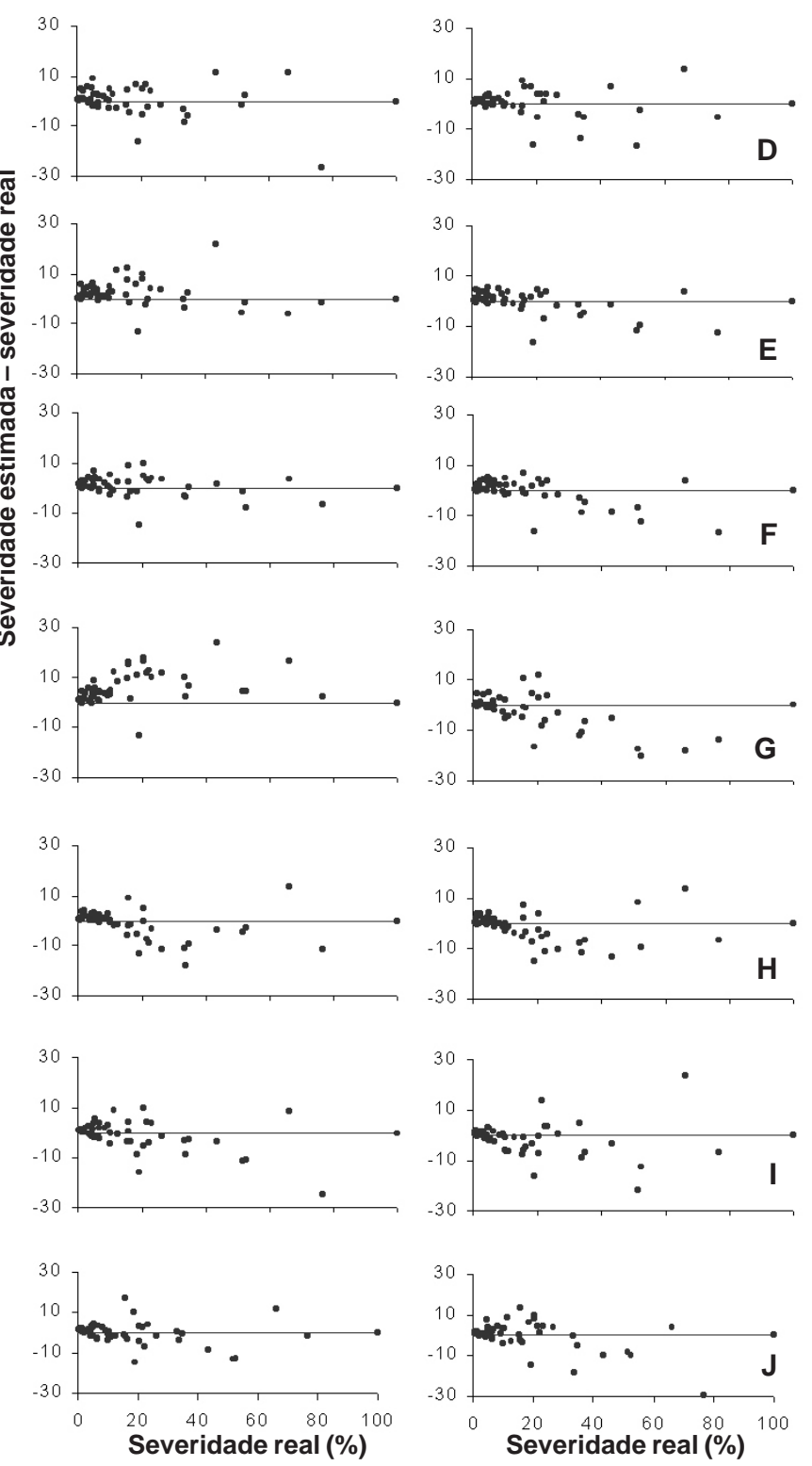

FIG. 3 - Resíduos (•) das análises de regressão linear entre valores de severidade real e estimada, com o auxílio da escala diagramática para mancha-de-cercospora em melancia (Citrullus lanatus), na primeira (AV 1) e segunda (AV 2) avaliação, pelos dez avaliadores (A-J). 


\section{REFERÊNCIAS BIBLIOGRÁFICAS}

ATHAYDE SOBRINHO, C. \& MENEZES, M. Cercospora citrullina causando queima em folhas de melancia no estado do Piauí. Fitopatologia Brasileira 22:245. 1997. (Resumo).

CROUS, P.W. \& BRAUN, U. Mycosphaerella and its Anamorphs: 1. Names published in Cercospora and Passalora. Utrecht. Centraalbureau voor Schimmelcultures. 2003.

DÍAZ, C.G., BASSANEZI, R.B. \& BERGAMIN FILHO, A. Desenvolvimento e validação de uma escala diagramática para Xanthomonas axonopodis pv. phaseoli em feijoeiro. Summa Phytopathologica 27:35-39. 2001.

HALFELD-VIEIRA, B.A., NECHET, K.L. \& PEREIRA, P.R.V.S. Mancha-de-cercospora em melancia no estado de Roraima. Fitopatologia Brasileira 29:228. 2004a.

HALFELD-VIEIRA, B.A., NECHET, K.L., SILVA, M.A.S. \& BARBOSA, R.N.T. Incidência de doenças fúngicas em cucurbitáceas no estado de Roraima. Resumos Expandidos, 44을 Congresso Brasileiro de Olericultura, Campo Grande, MS. 2004b. pp.1-4.

HORSFALL, J.G. \& COWLING, E.B. Pathometry: the measurement of plant disease. In: Horsfall, J.G. \& Cowling, E.B. (Eds.) Plant Disease: an advanced treatise-how disease develops in populations. v.2, New York: Academic Press. 1978. pp.119-136.

KRANZ, J. Measuring plant disease. In: Kranz, J. \& Rotem, J. (Eds.) Experimental Techniques in Plant Disease Epidemiology. Heidelberg: Springer-Verlag. 1988. pp.35-50.

KUROZAWA, C., PAVAN, M.A. \& REZENDE, J.A.M. Doenças das cucurbitáceas. In: Kimati, H., Amorim, L., Rezende; J.A.M., Bergamin Filho, A. \& Camargo, L.E.A. (Eds.) Manual de
Fitopatologia: doenças das plantas cultivadas. v.2. 4 ed. São Paulo: Editora Ceres. 2005. pp.293-302.

LEITE, R.M.V.B.C. \& AMORIM, L. Elaboração e validação de escala diagramática para mancha de Alternaria em girassol. Summa Phytopathologica: 28:14-19. 2002.

MICHEREFF, S.J., MAFFIA, L.A. \& NORONHA, M.A. Escala diagramática para avaliação da severidade da queima das folhas do inhame. Fitopatologia Brasileira 25:612-619. 2000.

NUTTER Jr., F.W., TENG, P.S. \& SHOKES, F.M. Disease assessment terms and concepts. Plant Disease 75:1187-1188. 1991.

NUTTER Jr., F.W., GLEASON, M.L., JENCO, J.H. \& CHRISTIANS, N.C. Assessing the accuracy, intra-rater repeatability, and inter-rater reliability of disease assessment systems. Phytopathology 83:806-812. 1993.

NUTTER Jr., F.W. \& SCHULTZ, P.M. Improving the accuracy and precision of disease assessments: selection of methods and use of computer-aided trainning programs. Canadian Journal of Plant Pathology 17:174-184. 1995.

PARKER, S.R., SHAW, M.W. \& ROYLE, D.J. The reliability of visual estimates of disease severity on cereal leaves. Plant Pathology 43:856-865. 1995.

RÊGO, A.M. \& CARRIJO, I.V. Doenças das cucurbitáceas. In: Zambolim, L., Vale, F.X.R. \& Costa, H. (Eds.) Controle de Doenças de Plantas: hortaliças. v.2. Visconde do Rio Branco: Suprema gráfica e editora. 2000. pp.535-597.

SPÓSITO, M.B., AMORIM, L., BELASQUE JUNIOR, J., BASSANEZI, R.B. \& AQUINO, R. Elaboração e validação de escala diagramática para avaliação da severidade da mancha preta em frutos cítricos. Fitopatologia Brasileira 1:81-85. 2004. 